\title{
Correction to: Long time dynamics for semi-relativistic NLS and half wave in arbitrary dimension
}

\author{
Jacopo Bellazzini ${ }^{1}$ - Vladimir Georgiev ${ }^{2,3,4} \cdot$ Nicola Visciglia $^{2}$
}

Published online: 16 March 2020

๑) Springer-Verlag GmbH Germany, part of Springer Nature 2020

\section{Correction to: Math. Ann. (2018) 371:707-740 https://doi.org/10.1007/s00208-018-1666-z}

The assumptions of Theorem 1.2 are not correct in case $n=1$. In dimension $n=1$, instead of $1+\frac{2}{n}<p<1+\frac{2}{n-1}$ it is correct $3<p<5$. Indeed the scaling argument in Proposition 3.1 works only if $3<p<5$. The correct Theorem is now the following:

Theorem 1.2 Let $3<p<5$ if $n=1$ and $1+\frac{2}{n}<p<1+\frac{2}{n-1}$ if $n \geq 2$. There exists $r_{0}>0$ such that the following conditions occur for every $r \in\left(0, r_{0}\right)$ :

- $\mathcal{J}_{r}>-\infty, \mathcal{B}_{r} \neq \emptyset$ and $\mathcal{B}_{r} \subset B_{1 / 2} \cap H^{1}\left(\mathbb{R}^{n}\right)$, where

$$
\mathcal{B}_{r}:=\left\{v \in S_{r} \cap B_{1} \text { s.t. } \mathcal{E}_{s}(v)=\mathcal{J}_{r}\right\}
$$

In particular for every $v \in \mathcal{B}_{r}$ there exists $\omega \in \mathbb{R}$ such that

$$
\sqrt{1-\Delta} v+\omega v-v|v|^{p-1}=0
$$

The original article can be found online at https://doi.org/10.1007/s00208-018-1666-z.

$凶$ Nicola Visciglia

viscigli@dm.unipi.it

Jacopo Bellazzini

jbellazzini@uniss.it

Vladimir Georgiev

georgiev@dm.unipi.it

1 Università di Sassari, Via Piandanna 4, 70100 Sassari, Italy

2 Dipartimento di Matematica, Università di Pisa, Largo B. Pontecorvo 5, 56100 Pisa, Italy

3 Faculty of Science and Engineering, Waseda University, 3-4-1, Okubo, Shinjuku-ku, Tokyo 169-8555, Japan

4 IMI-BAS, Acad. Georgi Bonchev Str., Block 8, 1113 Sofia, Bulgaria 
- the elements in $\mathcal{B}_{r}$ are ground states on $S_{r}$, namely:

$$
\inf _{\mathcal{C}_{r}} \mathcal{E} s(w)=\mathcal{J}_{r} \text { where } \mathcal{C}_{r}=\left\{w \in S_{r} \text { s.t. } \mathcal{E}_{s}^{\prime} \mid S_{r}(w)=0\right\}
$$

Assume moreover the following assumption:

$$
\sup _{\left(-T_{-}(f), T+(f)\right)}\|u(t, x)\|_{H^{1 / 2}\left(\mathbb{R}^{n}\right)}<\infty \Rightarrow T_{ \pm}(f)=\infty
$$

where $\left(-T_{-}(f), T+(f)\right)$ is the maximal time of existence of $u(t, x)$ which is the nonlinear solution to $s N L S$ with initial datum $f(x) \in H_{\text {rad }}^{1}\left(\mathbb{R}^{n}\right)$. Then we get:

- the set $\mathcal{B} \cap \cap H_{\text {rad }}^{1 / 2}\left(\mathbb{R}^{n}\right)$ is weakly orbitally stable for the flow associated with $s N L S$.

We warmly thank Louis Jeanjean for pointing out this mistake.

Publisher's Note Springer Nature remains neutral with regard to jurisdictional claims in published maps and institutional affiliations. 